\title{
WiRe57 : A Fine-Grained Benchmark for Open Information Extraction
}

\author{
William Léchelle, Fabrizio Gotti, Philippe Langlais \\ RALI, University of Montreal \\ \{lechellw, gottif, felipe\} diro.umontreal.ca
}

\begin{abstract}
We build a reference for the task of Open Information Extraction, on five documents. We tentatively resolve a number of issues that arise, including coreference and granularity, and we take steps toward addressing inference, a significant problem. We seek to better pinpoint the requirements for the task. We produce our annotation guidelines specifying what is correct to extract and what is not. In turn, we use this reference to score existing Open IE systems. We address the nontrivial problem of evaluating the extractions produced by systems against the reference tuples, and share our evaluation script. Among seven compared extractors, we find the MinIE system to perform best.
\end{abstract}

\section{Introduction}

Open Information Extraction systems, starting with TextRunner (Yates et al., 2007), seek to extract all relational tuples expressed in text, without being bound to an anticipated list of predicates. Such systems have been used recently for relation extraction (Soderland et al., 2013), questionanswering (Fader et al., 2014), and for building domain-targeted knowledge bases (Mishra et al., 2017), among others.

Subsequent extractors (ReVerb, Ollie, ClausIE, OpenIE 4, etc.) have sought to improve yield and precision, i.e. the number of facts extracted from a given corpus, and the proportion of those facts that is deemed correct.

Nonetheless, the task definition is underspecified, and, to the best of our knowledge, there is no gold standard. Most evaluations require somewhat subjective and inconsistent judgment calls to be made about extracted tuples being acceptable or not. The most recent automatic benchmark of Stanovsky and Dagan (2016) has some shortcomings that we propose to tackle here, regarding the theory underlining the task definition as well as the evaluation procedure.

We manually performed the task of Open Information Extraction on 5 short documents, elaborating tentative guidelines for the task, and resulting in a ground truth reference of 347 tuples. We evaluate against our benchmark the available OIE engines up to MinIE, with a fine-grained token-level evaluation. We distribute our resource and annotation guidelines, along with the evaluation script. ${ }^{1}$

\section{Related Work}

For their evaluation, typically, developers of Open IE systems pool the output of various systems on a given corpus. They label a sample of produced tuples as correct or incorrect, with the general guideline that an extraction is correct if it is implied by the sentence. Thus, Mausam et al. (2012) write: "Two annotators tagged the extractions as correct if the sentence asserted or implied that the relation was true." Del Corro and Gemulla (2013) propose: "We also asked labelers to be liberal with respect to coreference or entity resolution; e.g., a proposition such as ('he'; 'has'; 'office'), or any unlemmatized version thereof, is treated as correct." Saha et al. (2017): "We sample a random testset of 2,000 sentences [...] Two annotators with NLP experience annotate each extraction for correctness." Gashteovski et al. (2017): "A triple is labeled as correct if it is entailed by its corresponding clause." Then, precision and yield are used as performance metrics. Without a reference, recall is naturally impossible to measure.

We define a reference a priori. This allows for automatic scoring of systems' outputs, which greatly diminishes subjectivity from the process of labelling facts "for correctness". Above all, it is meant to help researchers agree on what the task

\footnotetext{
${ }^{1}$ https://github.com/rali-udem/WiRe 57
} 
precisely entails. Therefore, it allows to measure a true recall (albeit on a small corpus).

The complexity of our guidelines is indicative of all that is swept under the carpet when "annotating for correctness". As a matter of fact, when closely examining other references for OIE, many extracted tuples eventually labelled as "good" have more or less important issues. Some really dubious cases are hard to gauge and their labelling is ultimately subjective. To showcase the devilishly difficult judgment calls that this implies, compare the following two extractions. "The opportunity is significant and I hope we can take the opportunity to move forward,' he said referring to his coming trip to Britain." yields (his ; has ; coming trip), and "[...], the companies included CNN, but not its parent, AOL Time Warner" yields (its ; has ; parent). Are the extractions implied by the sentence ? In (Del Corro and Gemulla, 2013), the annotator approved the latter, and rejected the former. The extraction (he ; said ; The opportunity is significant referring to his coming trip to Britain) was also deemed correct, despite the composed second argument.

Some other tasks for which OIE output is used, such as Open QA (Fader et al., 2014), TAC-KBP (Soderland et al., 2013), or textual similarity and reading comprehension as in (Stanovsky et al., 2015) - could in principle be used to compare extractors' performance, but only give a very coarsegrained signal, mostly unaffected by the tuning of systems.

A promising method is that explored by Mishra et al. (2017) for the Aristo KB. ${ }^{2}$ Aristo is a science-focused KB extracted from a high-quality $7 \mathrm{M}$-sentence corpus. The authors preprocessed a smaller, similarly science-related, independent corpus of $1.2 \mathrm{M}$ sentences, into a "Reference KB" of 4147 facts, validated by Turkers. Assuming that these 4147 facts are representative of the science domain as a whole, they measured comprehensiveness (recall) over this domain by measuring coverage on the Reference KB.

\subsection{ORE benchmark}

Mesquita et al. (2013) compare more or less deep 'parsers', including the OIE systems Ollie and ReVerb, on the germane task of Open Relation Extraction (ORE), between named entities. They build a benchmark of 662 binary relations over

\footnotetext{
${ }^{2}$ http://data.allenai.org/tuple-kb/
}

1100 sentences from 3 sources (the Web, the New York Times and the Penn Treebank). They label an additional 222 NYT sentences with as many $n$-ary relations, and 12,000 with automatic annotations.

Besides the named entity arguments, their annotations consist of one mandatory trigger word (indicating the relation), surrounded by a window of allowed tokens. To compare OIE with ORE systems, they have to replace the target entities by salient arguments (Asia and Europe) which are easy to recognize. They discuss some of the challenges that arise from divergent annotation styles and evaluation methods.

While the tasks are similar, restraining arguments to be named entities limits IE to capturing only the most salient relations expressed in the text. Allowing for any NP to be an argument, we extract 6 facts per sentence on average in the benchmark presented here, compared to 0.6 in the ORE dataset. We also annotate some relations that do not have a trigger in the sentence (such as (Paris ; [is in] ; France) from "Chilly Gonzales lived in Paris, France").

\subsection{QA-SRL OIE benchmark}

Stanovsky and Dagan (2016) build a large benchmark for OIE, by automatically processing the QA-SRL dataset (He et al., 2015). Precisely, for each predicate annotated in QA-SRL, they generate one tuple expressing each element of the Cartesian product of answers to the questions about this predicate.

For instance, QA-SRL lists five questions asked about the sentence "Investors are appealing to the SEC not to limit their access to information about stock purchases and sales by corporate insiders": "who are appealing to something ?", "who are someone appealing to ?", "what are someone appealing ?", "what might not limit something ?" and "what might not someone limit ?", with one answer per question. This generates the reference tuples (Investors ; appealing ; not to limit their access to information about stock purchases and sales by corporate insiders; to the SEC) and (the $S E C$; might not limit ; their access to information about stock purchases and sales by corporate insiders).

Their dataset is comprised of 10,359 tuples over 3200 sentences (from the Wall Street Journal and Wikipedia), and is available for download. ${ }^{3}$

\footnotetext{
${ }^{3}$ http://u.cs.biu.ac.il/ nlp/resources/
} 
While this work makes a big step in the right direction, there are a few important issues with this benchmark.

First, a major strength of the dataset is its intended and partly achieved completeness, but we do not find it to be a suitably comprehensive reference against which to measure systems' recall. This might be because the QA-SRL dataset doesn't lend itself well to exhaustiveness in the realm of Open IE, partly because it is restricted to explicit predicates. For instance, the sentence “However, Paul Johanson, Monsanto's director of plant sciences, said the company's chemical spray overcomes these problems and is 'gentle on the female organ'." contains two predicates, generating the extractions (Paul Johanson; said; the company's chemical spray overcomes these problems and is "gentle on the female organ.") and (the company's chemical spray; overcomes; these problems). Yet, that omits the (in our view useful) extractions (the company's chemical spray ; is ; "gentle on the female organ"), and (Paul Johanson ; is ; Monsanto's director of plant sciences).

Another issue is that some words not found in the original sentence were quietly added by the SRL-to-QA process, retained in the QA-to-OIE transformation, and become part of the reference. In the example above, it is unclear how the second predicate "might not limit" is extracted from the sentence. At the very least, the fact that these words are foreign to the original sentence should be made explicit. Further, although in this particular case adding the modal is a good way of expressing the information, its repeated use by QASRL to produce questions waters down the expressed facts in the end. For instance, the uninformative triple (a manufacturer ; might get ; something) is generated from the sentence "... and if a manufacturer is clearly trying to get something out of it ...", with the same added "might".

Last, the scoring procedure is not robust. Using the code made available by the authors ${ }^{4}$, we were able to get top results with a dummy extractor.

This is because the scorer doesn't penalize extractions for being too long, nor for misplacing parts of the relation in the object slot or vice versa. Therefore, if $w_{0} w_{1} \ldots w_{n}$ is an input sentence, a

${ }^{4}$ https: / / github.com/gabrielstanovsky/ oie-benchmark - the scoring function was updated since its description in the article. We believe the published function suffers from similar issues.
}

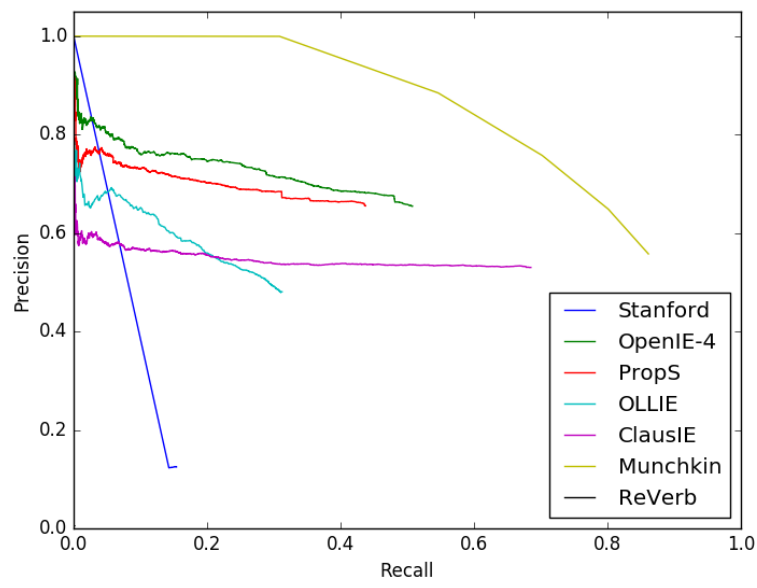

Figure 1: Performance metrics must take span precision into account. The 25-line long Munchkin script returns variations of the full sentence (with decreasing confidence) and is not penalized by the evaluation script of the latest benchmark (Stanovsky and Dagan, 2016). Its superior performance is artificially inflated.

trivial system that "extracts" $\left(w_{0} ; w_{1} ; w_{2} \ldots w_{n}\right)$, $\left(w_{0} ; w_{1} w_{2} ; w_{3} \ldots w_{n}\right)$, etc., will be given an unfairly great score. We implemented that program (dubbed Munchkin) which predictably performed well above other genuine extraction systems, as pictured in Figure 1.

\subsection{RelVis benchmarking toolkit}

Schneider et al. (2017) evaluate four systems (ClausIE, OpenIE 4, Stanford Open IE and PredPatt) against the two datasets mentioned above. ${ }^{5}$ They use two methods to match predicted and reference tuples : "containment" and "relaxed containment". These methods mean that the predicted tuple must include the reference tuple, and that inclusion must happen for each argument, in the non-relaxed case. In the relaxed case, the boundaries between parts of a tuple are ignored. Like that of Stanovsky and Dagan (2016), this scoring procedure doesn't penalize systems for returning overlong spans.

\subsection{Scoring}

To compare facts with a reference, most authors require matching tuples to have the same number of arguments and to share the grammatical head words of each part, e.g. Angeli and Manning (2013) and the article of Stanovsky and Da-

\footnotetext{
${ }^{5}$ Their code is announced but not available as of this writing - https://github.com/schmaR/relvis
} 
gan (2016). In their updated GitHub repository, Stanovsky and Dagan (2016) instead use lexical match : more than half of the words of a predicted tuple must match the reference for it to be correct.

In contrast with these works and (Schneider et al., 2017), our scorer penalizes verbosity by measuring precision at the token level. We penalize the omission of parts of a reference tuple by gradually diminishing recall (at the token level), instead of a sharp all-or-nothing criterion.

Mesquita et al. (2013) annotate relations as one mandatory target plus some optional complementary words, and treat arguments (named entities) in an ad-hoc fashion for OIE systems.

\section{$3 \quad$ WiRe57}

Open IE bears some similarity to the task of Semantic Role Labelling, as explored in (Christensen et al., 2011; Mesquita et al., 2013), and as demonstrated by SRLIE, a component of OpenIE 4.

In effect extracted tuples are akin to simplified PropBank ${ }^{6}$ or FrameNet ${ }^{7}$ frames, and our annotations were inspired by those projects. Still, with a focus on extracting new relations at scale, optional arguments such as Propbank's modifiers (ArgM) are discouraged in OIE. Another major difference is the vocabulary of predicates being open to any relational phrase, rather than belonging to a closed curated list such as VerbNet. Within reason, OIE seeks to extract rich and precise relations phrases.

\begin{tabular}{lrr} 
Phenomenon & $\mathrm{N}$ & $\%$ \\
\hline All tuples & 343 & 100 \\
Anaphora & 196 & 57 \\
Contains inferred words & 186 & 54 \\
Hallucinated parts & 135 & 39 \\
Binary relations & 254 & 74 \\
$n$-ary, $n=3$ & 72 & 21 \\
$n$-ary, $n=4$ & 16 & 5 \\
$n$-ary, $n=5$ & 1 & 0.3 \\
\hline Inferred words & $347 / 2597$ & 13.4
\end{tabular}

Table 1: Frequencies of various phenomena in WiRe57.

\subsection{Annotation process}

A small corpus of 57 sentences taken from the beginning of 5 documents in English was used as the

\footnotetext{
${ }^{6}$ propbank.github.io - (Kingsbury and Palmer, 2002)

${ }^{7}$ framenet.icsi.berkeley.edu - (Ruppenhofer et al., 2005)
}

source text from which to extract tuples. Three documents are Wikipedia articles (Chilly Gonzales, the EM algorithm, and Tokyo) and two are newswire articles (taken from Reuters, hence the Wi-Re name).

Two annotators (authors of this paper) first independently extracted tuples from the documents, based on a first version of the annotation guidelines which quickly proved insufficient to reach any significant agreement. The two sets of annotations were then merged, and the guidelines rectified along the way in order to resolve the issues that arose. After merging, a quick test on a few additional sentences from a different document showed a much improved agreement, more than half of extractions matching exactly and the remaining missing a few details. The guidelines are detailed in the next sections.

\subsection{Annotation principles}

In keeping with past literature, our guiding principles for the annotation were as follows.

The first, obvious purpose of extracted information is to be informative. Fader et al. (2011) mention how extracting (Faust ; made ; a deal) instead of the correct (Faust; made a deal with; the devil) would be pointless. Further, anaphoric mentions being so ubiquitous and being void of meaning outside the context of their original sentence, we resolve anaphora in our extractions.

Moreover and following (Stanovsky and Dagan, 2016), extracted tuples should each be minimal, in the sense that they should convey the smallest standalone piece of information, though that piece must be completely expressed. Thus, some facts must be extracted as $n$-ary relations. ${ }^{8}$ The MinIE system in particular addresses this issue and "minimizes its extractions by identifying and removing parts that are considered overly specific".

The annotation shall be exhaustive, in the sense of capturing as much of the information expressed in the text as possible. This is to measure absolute recall for a system, a notoriously difficult evaluation metric for Open IE.

This in turn raises the issue of inference: some information is merely suggested by the text, rather than explicitly expressed, and should not be annotated. Light inference, in the form of reformulation, is helpful to make use of the information ex-

\footnotetext{
${ }^{8}$ Some systems - namely CSD-IE (Bast and Haussmann, 2013) and NestIE (Bhutani et al., 2016) - explore nesting extractions, but we didn't adopt this strategy.
} 
tracted, but full-fledged inference should be processed by a dedicated program, and is not part of the Open IE task. Because the concept of "light inference" is subjective, we propose in the guidelines a few examples and counterexamples that delineate the limits between the two classes.

Other authors mention this issue. From (Wu and Weld, 2010) : "The extractor should produce one triple for every relation stated explicitly in the text, but is not required to infer implicit facts." Stanovsky and Dagan (2016) say: "an Open IE extractor should produce the tuple (John; managed to open; the door) but is not required to produce the extraction (John; opened; the door)". In our resource we do also annotate (John; [opened]; the door), marking the reworded relation as inferred (which in turn makes it optional to find when scoring).

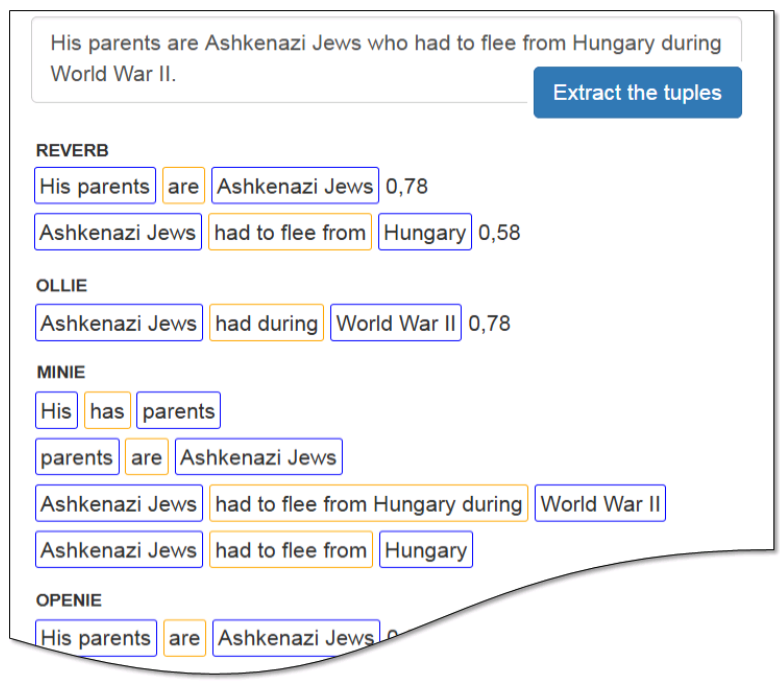

Figure 2: Example output of evaluated OIE systems, on sentence $\mathrm{CH}$ 7. This cropped screenshot is of a inhouse web application that allows us to submit any sentence for tuple extraction and to visualize the results.

\subsection{Annotation guidelines 9}

Extracted tuples should reflect all meaningful relationships found in the source text. Typically, this means that there are multiple tuples for a given sentence. A number of times, two arguments are connected in a sentence but the relation that links them is implicit (e.g. Paris, France; the North Atlantic Treaty Organization (NATO); the Nature paper or the Turing paper, etc.). In this case, we

\footnotetext{
${ }^{9}$ We share at https: / / github.com/rali-udem/ WiRe 57 our annotation guidelines. We present its major points here.
}

Sentence CH 7 - "His parents are Ashkenazi Jews who had to flee from Hungary during World War II."

\section{Annotations}

- (His/(Chilly Gonzales's) parents ; are ; Ashkenazi Jews)

- (His/(Chilly Gonzales's) parents ; are ; Jews)

- (His/(Chilly Gonzales's) parents ; had to flee from ; Hungary ; during World War II)

- (His/(Chilly Gonzales's) parents ; [fled] from ; Hungary ; during World War II)

- ([Chilly Gonzales] ; [has] ; parents)

Sentence EM 5 - "They pointed out that the method had been 'proposed many times in special circumstances' by earlier authors."

\section{Annotations}

- (They/(Arthur Dempster, Nan Laird, and Donald Rubin) ; pointed out that ;

(the method)/(The EM algorithm) had been "proposed many times in special circumstances" by earlier authors) - ((the method)/(The EM algorithm) ; had been proposed by; earlier authors ; in special circumstances) [attributed]

- (earlier authors ; proposed ; (the method)/(The EM algorithm); in special circumstances) [attributed]

Sentence FI 2 - "A police statement did not name the man in the boot, but in effect indicated the traveler was State Secretary Samuli Virtanen, who is also the deputy to Foreign Minister Timo Soini."

\section{Annotations}

- (A police/(Finnish police) statement ; did not name ; (the man in the boot)/(Samuli Virtanen))

- ((the man in the boot)/(Samuli Virtanen); was ; Samuli Virtanen) [attributed]

- ((the traveler)/(Samuli Virtanen) ; was ; Samuli Virtanen)

- (Samuli Virtanen ; [is] ; State Secretary) [attributed]

- (Samuli Virtanen ; is ; the deputy to Foreign Minister

- (Samuli Virtanen ; is ; [a] deputy)

- (Timo Soini ; [is] ; Foreign Minister)

- (Timo Soini ; [has] ; [a] deputy)

Sentence CE 4 - "The International Monetary Fund, for example, saw 2017 global growth at 3.4 percent with advanced economies advancing 1.8 percent."

\section{Annotations}

- (The International Monetary Fund ; saw ; 2017 global growth ; at 3.4 percent)

- (The International Monetary Fund ; saw ; advanced economies ; advancing 1.8 percent ; [in] 2017)

- (2017 global growth ; [was] ; 3.4 percent)

- (advanced economies ; [advanced] ; 1.8 percent ;

[in] 2017) [attributed]

Figure 3: Sample annotations from WiRe57, from four of the documents. Reformulated words are enclosed in [brackets] and coreference information is indicated with forward slashes and parentheses.

annotate a somewhat arbitrary relationship (such as is in, stands for, published in and published by respectively), the tokens of which are thus inferred. This is the case for $39 \%$ of our tuples.

Some OIE systems similarly attempt to halluci- 
nate some or part of relations. Notably, ClausIE wrongly extracts (New Delhi ; is ; India), and MinIE gets right (Paris ; is in ; France). Ollie adds some "be" auxiliaries to otherwise nominal relations, as in Barack Obama, former president of the United States, [...], which OpenIE 4 also infers. Yet, we acknowledge that most work in Open IE rely on explicit predicate tokens as in (Mesquita et al., 2013), and don't try to elicit relations further. At scoring time, systems are not penalized for not finding inferred words, or not finding inferred relations. If the whole predicate of a tuple is inferred, a predicted tuple is scored on its token overlap with the arguments only.

We suggest "platinum" annotations, including inferred words, to be a very high standard for extractors, while the gold standard for the task, recall-wise, is based only on words found in the original sentences.

Noun phrases can be rich in elements of information. To solve the problem of finding the granularity level to use when including argument NPs, we extract two tuples, one as generic as possible and the other as specific as possible, for the same relation. Adjectives and other elements of meaning that can be easily separated from the noun phrase to create other tuples are so split. Only elements that cannot be separated become part of the most specific noun phrase.

For instance, the sentence "Solo Piano is a great album of classical piano compositions" would yield 3 tuples : the split adjective (Solo Piano ; is ; great), the generic (Solo Piano ; is ; [an] album) and the specific (Solo Piano ; is ; [an] album of classical piano compositions).

When predicates contain nouns or other elements (e.g. Tokyo is the capital of Japan.), we annotate the richer relationship (Tokyo ; is the capital of ; Japan) rather than the more basic (Tokyo ; is ; the capital of Japan). This allows tuple relations to be more meaningful, and more easily compared, clustered, and aggregated with other relations. This also is in line with ReVerb.

Like ClausIE and other extractors since, we split conjunctions: "Andrea lived in both Poland and Italy" yields both (Andrea ; lived in ; Poland) and (Andrea ; lived in ; Italy).

\subsection{Resource}

A sample of annotations is pictured in Figure 3. The occurring frequency of various phenomena is presented in Table 1. Our resource is comprised of 343 relational facts (or tuples), three quarters of them binary relations. One in five have three arguments, sometimes "two objects" as in (This performance; has made; some economists ; optimistic) or more frequently a complement as in (His parents ; had to flee; from Hungary ; during World War II). Five percent of them have four arguments or more : for instance (Tokyo ; ranked ; third ; in the International Financial Centres Development IndexEdit ; twice) and (The International Monetary Fund ; saw ; advanced economies ; advancing 1.8 percent ; [in] 2017).

We found (and resolved) anaphoric phrases in more than half the tuples, as in (Emperor Meiji ; moved ; his/(Emperor Meiji's) seat ; to (the city)/Tokyo ; from the old capital of Kyoto ; in 1868). The released dataset contains the raw and anaphora-resolved argument spans.

When solely extracting words from the sentence would not yield clear factual tuples, we reworded or adapted the text into more explicit statements. In this case, we explicitly marked the changed (or added) words as inferred (they are bracketed in Figure 3). For instance in sentence CE 4, the relation "[advanced]" was reformulated from the sentence word "advancing", and the word [in] was added before "2017". In the resource, each token is accompanied by its index in the sentence if it comes from it, or the "inferred" mark. Inferred words represent $13 \%$ of the lot but affect $54 \%$ of the tuples.

\subsection{Inter-annotator agreement}

\begin{tabular}{lrrrr} 
& \# tokens & $1 \leftrightarrow 2$ & $1 \leftrightarrow \mathrm{R}$ & $2 \leftrightarrow \mathrm{R}$ \\
\hline Sentence 1 & 24 & 84.4 & 90.6 & 93.8 \\
Sentence 2 & 19 & 98.7 & 98.7 & 100 \\
Sentence 3 & 33 & 78.0 & 90.9 & 85.6 \\
\hline Average & & $\mathbf{8 5 . 2}$ & $\mathbf{9 2 . 8}$ & $\mathbf{9 1 . 9}$
\end{tabular}

Table 2: Inter-annotator agreement. Percentage of agreement on the labelling of each sentence token as belonging to 4 classes. Each annotator's original production differs only slightly from the agreed-on result (columns $1 \leftrightarrow \mathrm{R}$ and $2 \leftrightarrow \mathrm{R}$ ), and the disagreement between both annotators is slightly larger (column $1 \leftrightarrow 2$ ). The average is computed token-wise.

As mentioned in section 3.1, a qualitatively high agreement was reached after the merging of preliminary annotations and deliberation over the guidelines' items. After the guidelines were fully 
settled, three additional sentences from one of the documents were annotated by two annotators (1 and 2) in order to quantitatively measure interannotator agreement. Afterward, annotation discrepancies were resolved in cases of disagreement to produce a merged reference (R). Here, we report the agreement between the two original annotations $(1 \leftrightarrow 2)$, and between each original annotation and the merged reference $(1 \leftrightarrow R$ and $2 \leftrightarrow R)$.

Comparing triples can become quite tricky for many reasons, including missing complements, overlapping spans, etc. We therefore resorted to another scheme, where we reframe the annotation task as taking each annotated token and classifying it as either belonging or not belonging to each of 4 classes (subject, relation, object, or complementary argument). These classifications can be trivially derived from the triples produced beforehand. For instance, a triple $\left(t_{1} t_{2} ; t_{3} ; t_{4} t_{5}\right)$ implies that the annotator classified tokens $t_{1}$ and $t_{2}$ as belonging to the subject class. It then becomes possible to measure an agreement percentage on the full binary labelling grid (obtained automatically from the long-form annotations). We believe the resulting figures (shown in Table 2) aptly reflect the level of overall agreement between the annotators, despite the minimal sample size. We measure an overall inter-annotator agreement $(1 \leftrightarrow 2)$ of $85.2 \%$ for the three sentences.

Qualitatively, one annotator steered close to the sentence syntax, sometimes missing some of the meaning obscured by long-winded formulations. The other annotator tended to be overly specific, including some non-essential complements, and making longer-ranged inferences that fall out of the scope of this task. Some possessive and passive constructions were also overlooked.

\section{Evaluation of Existing Systems}

\subsection{Scorer}

An important step when measuring extractors' performances is the scoring process. Matching a system's output to a reference is not trivial. As detailed in Section 2.2, because it didn't penalize overlong extractions, we could game the basic evaluation method of the QA-SRL OIE benchmark with a trivial extractor.

Our scorer computes precision and recall of a system's predicted tuples at the token level. Precision is, briefly put, the proportion of extracted words that are found in the reference. Recall is the proportion of reference words found in the systems' predictions.

More formally, let $G=\left\{g_{1}, g_{2}, \ldots, g_{N}\right\}$ be the gold tuples, and $T_{\text {sys }}=\left\{t_{1}, t_{2}, \ldots, t_{n}\right\}$ a system's extractions. We denote the parts of a tuple $t=$ $\left(t^{a_{1}} ; t^{r} ; t^{a_{2}} ; t^{a_{3}} ; \ldots\right)=\left(t^{p_{k}}\right)_{k \in[1,6]}$, where $p_{1}$ is the first argument, $p_{2}$ is the relation, etc., up to $p_{6}$ the fifth argument when it exists (no reference tuple contains more than 5 arguments). Let $t_{i}^{p} \cap g_{j}^{p}$ be the subset of words shared by parts $t_{i}^{p}$ and $g_{j}^{p}$, where parts are considered as bags of words. The length of a tuple is the sum of lengths of its parts, i.e. $\left|t_{i}\right|=\left|t_{i}^{a_{1}}\right|+\left|t_{i}^{r}\right|+\left|t_{i}^{a_{2}}\right|+\left|t_{i}^{a_{3}}\right|+\cdots=\sum_{k}\left|t_{i}^{p_{k}}\right|$.

A predicted tuple $t_{i}$ may match a reference tuple $g_{j}$ from the same sentence if they share at least one word from each of the relation, first and second arguments, that is iff $\left(w_{a_{1}}, w_{r}, w_{a_{2}}\right)$ exist such that $w_{1} \in g_{j}^{a_{1}} \cap t_{i}^{a_{1}}, w_{r} \in g_{j}^{r} \cap t_{i}^{r}$ and $w_{2} \in g_{j}^{a_{2}} \cap t_{i}^{a_{2}}$.

For all tuple pairs that may match, we have the matching scores:

$$
\begin{aligned}
\operatorname{precision}\left(t_{i}, g_{j}\right) & =\frac{\sum_{k}\left|t_{i}^{p_{k}} \cap g_{j}^{p_{k}}\right|}{\left|t_{i}\right|} \\
\operatorname{recall}\left(t_{i}, g_{j}\right) & =\frac{\sum_{k}\left|t_{i}^{p_{k}} \cap g_{j}^{p_{k}}\right|}{\left|g_{j}\right|} \\
F_{1} & =\frac{2 p r}{p+r} .
\end{aligned}
$$

We match predicted tuples with reference ones by greedily removing from the potential match pool the pair with maximum $F_{1}$ score, until no remaining tuples match. Let $m($.$) be the match-$ ing function such that $t_{i}$ matches with $g_{m(i)}$ (and conversely $t_{m(j)}$ matches $g_{j}$ ), assuming that $\mid t_{i} \cap$ $g_{m(i)} \mid=0$ if there is no match for $t_{i}$.

Hence, the overall performance metrics of an extractor are its token-weighted precision and recall over all tuples, i.e.

$$
\begin{aligned}
\operatorname{precision}_{\mathrm{sys}} & =\frac{\sum_{i}^{n}\left(\sum_{k}\left|t_{i}^{p_{k}} \cap g_{m(i)}^{p_{k}}\right|\right)}{\sum_{i}^{n}\left|t_{i}\right|} \\
\text { recall }_{\mathrm{sys}} & =\frac{\sum_{j}^{N}\left(\sum_{k}\left|t_{m(j)}^{p_{k}} \cap g_{j}^{p_{k}}\right|\right)}{\sum_{j}^{N}\left|g_{j}\right|} \\
F_{1_{\mathrm{sys}}} & =\frac{2 p_{\mathrm{sys}} r_{\mathrm{sys}}}{p_{\mathrm{sys}}+r_{\mathrm{sys}}}
\end{aligned}
$$

To avoid penalizing systems for not finding them, neither the words annotated as inferred, nor 


\begin{tabular}{l|ccc|cc|ccc} 
& Extractions & Matches & $\begin{array}{c}\text { Exact } \\
\text { matches }\end{array}$ & $\begin{array}{l}\text { Prec. of } \\
\text { matches }\end{array}$ & $\begin{array}{c}\text { Recall of } \\
\text { matches }\end{array}$ & Prec. & Recall & F1 \\
\hline ReVerb (Fader et al., 2011) & 79 & 54 & 13 & .83 & .77 & $\mathbf{. 5 6 9}$ & .121 & .200 \\
Ollie (Mausam et al., 2012) & 145 & 74 & 8 & .73 & .81 & .347 & .175 & .239 \\
ClausIE (Del Corro and Gemulla, 2013) & 223 & 121 & $\mathbf{2 4}$ & .74 & .84 & .401 & .298 & .342 \\
Stanford (Angeli et al., 2015) & 371 & 99 & 2 & .79 & .65 & .210 & .188 & .198 \\
OpenIE 4 (Mausam, 2016) & 101 & 74 & 5 & .68 & .84 & .501 & .182 & .267 \\
PropS (Stanovsky et al., 2016) & 184 & 69 & 0 & .59 & .80 & .222 & .162 & .187 \\
MinIE (Gashteovski et al., 2017) & 252 & $\mathbf{1 3 4}$ & 10 & .75 & .83 & .400 & $\mathbf{. 3 2 3}$ & $\mathbf{. 3 5 8}$
\end{tabular}

Table 3: Performance of available OpenIE systems (in chronological order) on our reference. Precision and recall are computed at the token level. Systems with lower precision of matches are penalized for producing overlong tuples. High precision and recall of matches overall show that our matching function (one shared word in each of the first three parts) works correctly. Inferred words are required for exact matches.

the coreference information are used in this evaluation $\left(g_{j}\right.$ is the non-resolved version of the tuple, and inferred words are not included in recall denominators). Future work can look into evaluating OIE systems that mean to resolve anaphoras.

\subsection{Results}

In order to experiment with the 7 systems used in this paper, we bundled them as a web service. A client application need only submit a sentence and a list of OIE system names to perform extraction. All tuples are in turn served as uniform JSON objects, no matter the OIE system used. This facilitates the development of clients, shielded from the various tuple formats, coding languages, and other quirks of the OIE systems. It also allowed us to visualize the tuples using a web application (see Figure 2). Moreover, because the various extractors run as servers, they load their respective resources only once, when the service is launched, and are then always quick to respond to a given extraction task (a few seconds). Otherwise, the user would have had to wait a few minutes for the resources to load each time when querying the extractors.

While creating such a framework is a significant effort, it ultimately saved us a lot of time when writing the clients. It also provided a common frame of reference for all collaborators in our lab. Typically, we used the default configuration for each OIE system, but we tweaked the available flags in order to favor exhaustiveness, when such flags were present and properly documented. When additional information did not fit into a traditional tuple ( $\arg 1$; rel; $\arg 2)$, e.g. MinIE's quantities, we resorted to simple schemes to faithfully cast that information into a tuple.

Table 3 details the performance of available OIE systems against our reference. MinIE produces a large number of correct tuples, and performs best, especially recall-wise. The conservative choices made by ReVerb achieve a relatively high precision, though it lacks in comprehensiveness. Ollie improves recall over ReVerb, and Open IE 4 improves precision over Ollie. Stanford Open IE produces a very large number of tuples, hindering its precision (it is possible that limiting its verboseness through configuration would improve this).

\section{Conclusion}

In this paper, we set out to create additional resources useful to researchers in Open Information Extraction. We distribute these resources freely.

Primarily, we provide a manually crafted, tentative reference for the task. It consists of 343 manually extracted facts, including some implicit relations, over 57 sentences. A quarter of them are $n$-ary relations and coreference information is included in over half of them. We believe that such a benchmark is valuable because it offers a common frame of reference allowing OIE systems to be tested and compared fairly, a task we carried out on 7 OIE systems. This also entailed the creation of a scoring algorithm and program, which we release along with the data. We assess the ReVerb, Ollie, ClausIE, Stanford Open IE, OpenIE 4, PropS, and MinIE systems against our reference, using a fine-grained token-level scorer. We find the MinIE system to perform best.

Naturally, such an annotation effort requires one to attempt to "pin down" the task of OIE by confronting real-life data. We provide guidelines that propose such a definition. While by no means definitive or exhaustive, these guidelines have at least the merit of being sufficiently 
clear to yield an annotated dataset with a reasonable inter-annotator agreement. At the same time, we believe they are not too overwrought, and rather invite further contributions by other researchers. The thorniest issues are the fine line between useful reformulation of information to a canonical form and ill-advised inference, and how to trim and annotate complex noun-phrase arguments. These difficulties can affect the manual annotation process, and, interestingly, are also likely to arise when building OIE systems, which is the ultimate goal in this research field after all.

\section{References}

Gabor Angeli and Christopher D. Manning. 2013. Philosophers are mortal: Inferring the truth of unseen facts. In Proceedings of the Seventeenth Conference on Computational Natural Language Learning, CoNLL 2013, Sofia, Bulgaria, August 8-9, 2013, pages 133-142. ACL.

Gabor Angeli, Melvin Johnson Premkumar, and Christopher D. Manning. 2015. Leveraging linguistic structure for open domain information extraction. In $A C L$.

Hannah Bast and Elmar Haussmann. 2013. Open information extraction via contextual sentence decomposition. In Semantic Computing (ICSC), 2013 IEEE Seventh International Conference on, pages 154159. IEEE.

Nikita Bhutani, H V Jagadish, and Dragomir Radev. 2016. Nested propositions in open information extraction. In Proceedings of the 2016 Conference on Empirical Methods in Natural Language Processing, pages 55-64. Association for Computational Linguistics.

Janara Christensen, Mausam, Stephen Soderland, and Oren Etzioni. 2011. An analysis of open information extraction based on semantic role labeling. In Proceedings of the Sixth International Conference on Knowledge Capture, K-CAP'11, pages 113-120, New York, NY, USA. ACM.

Luciano Del Corro and Rainer Gemulla. 2013. Clausie: Clause-based open information extraction. In Proceedings of the 22Nd International Conference on World Wide Web, WWW'13, pages 355-366, Republic and Canton of Geneva, Switzerland. International World Wide Web Conferences Steering Committee.

Anthony Fader, Stephen Soderland, and Oren Etzioni. 2011. Identifying relations for open information extraction. In Proceedings of the Conference on Empirical Methods in Natural Language Processing, EMNLP' 11, pages 1535-1545, Stroudsburg, PA, USA. Association for Computational Linguistics.
Anthony Fader, Luke Zettlemoyer, and Oren Etzioni. 2014. Open question answering over curated and extracted knowledge bases. In Proceedings of the 20th ACM SIGKDD International Conference on Knowledge Discovery and Data Mining, KDD '14, pages 1156-1165, New York, NY, USA. ACM.

Kiril Gashteovski, Rainer Gemulla, and Luciano Del Corro. 2017. Minie: Minimizing facts in open information extraction. In Proceedings of the 2017 Conference on Empirical Methods in Natural Language Processing, pages 2630-2640. Association for Computational Linguistics.

Luheng He, Mike Lewis, and Luke Zettlemoyer. 2015. Question-answer driven semantic role labeling: Using natural language to annotate natural language. In Proceedings of the 2015 Conference on Empirical Methods in Natural Language Processing, pages 643-653. Association for Computational Linguistics.

Paul Kingsbury and Martha Palmer. 2002. From treebank to propbank. In Language Resources and Evaluation.

Mausam, Michael Schmitz, Robert Bart, Stephen Soderland, and Oren Etzioni. 2012. Open language learning for information extraction. In Proceedings of Conference on Empirical Methods in Natural Language Processing and Computational Natural Language Learning (EMNLP-CONLL).

Mausam Mausam. 2016. Open information extraction systems and downstream applications. In Proceedings of the Twenty-Fifth International Joint Conference on Artificial Intelligence, IJCAI'16, pages 4074-4077. AAAI Press.

Filipe Mesquita, Jordan Schmidek, and Denilson Barbosa. 2013. Effectiveness and efficiency of open relation extraction. In Proceedings of the 2013 Conference on Empirical Methods in Natural Language Processing, pages 447-457. Association for Computational Linguistics.

Bhavana Dalvi Mishra, Niket Tandon, and Peter Clark. 2017. Domain-targeted, high precision knowledge extraction. TACL, 5:233-246.

Josef Ruppenhofer, Michael Ellsworth, Miriam R. L. Petruck, Christopher R. Johnson, and Jan Scheffczyk. 2005. FrameNet II: Extended theory and practice. Technical report, ICSI.

Swarnadeep Saha, Harinder Pal, and Mausam. 2017. Bootstrapping for numerical open IE. In Proceedings of the 55th Annual Meeting of the Association for Computational Linguistics, ACL 2017, Vancouver, Canada, July 30 - August 4, Volume 2: Short Papers, pages 317-323. Association for Computational Linguistics.

Rudolf Schneider, Tom Oberhauser, Tobias Klatt, Felix A. Gers, and Alexander Löser. 2017. Analysing errors of open information extraction systems. In 
Proceedings of the First Workshop on Building Linguistically Generalizable NLP Systems, pages 1118. Association for Computational Linguistics.

Stephen Soderland, John Gilmer, Robert Bart, Oren Etzioni, and Daniel S. Weld. 2013. Open information extraction to KBP relations in 3 hours. In Proceedings of the Sixth Text Analysis Conference, TAC 2013, Gaithersburg, Maryland, USA, November 1819, 2013. NIST.

Gabriel Stanovsky and Ido Dagan. 2016. Creating a large benchmark for open information extraction. In Proceedings of the 2016 Conference on Empirical Methods in Natural Language Processing (EMNLP), Austin, Texas. Association for Computational Linguistics.

Gabriel Stanovsky, Ido Dagan, and Mausam. 2015. Open ie as an intermediate structure for semantic tasks. In Proceedings of the 53rd Annual Meeting of the Association for Computational Linguistics and the 7th International Joint Conference on Natural Language Processing (Volume 2: Short Papers), pages 303-308, Beijing, China. Association for Computational Linguistics.

Gabriel Stanovsky, Jessica Ficler, Ido Dagan, and Yoav Goldberg. 2016. Getting more out of syntax with props. CoRR, abs/1603.01648.

Fei Wu and Daniel S. Weld. 2010. Open information extraction using wikipedia. In Proceedings of the 48th Annual Meeting of the Association for Computational Linguistics, ACL '10, pages 118-127, Stroudsburg, PA, USA. Association for Computational Linguistics.

Alexander Yates, Michael Cafarella, Michele Banko, Oren Etzioni, Matthew Broadhead, and Stephen Soderland. 2007. Textrunner: Open information extraction on the web. In Proceedings of $\mathrm{Hu}$ man Language Technologies: The Annual Conference of the North American Chapter of the Association for Computational Linguistics: Demonstrations, NAACL-Demonstrations '07, pages 25-26, Stroudsburg, PA, USA. Association for Computational Linguistics. 